\title{
Registro de sobrepeso y obesidad como problema de salud por pediatras de cabecera en una historia clínica electrónica
} Recording of overweight and obesity as a health problem by primary care pediatricians in an electronic medical record

\author{
Dra. Carolina Silva ${ }^{a}$ Dra. Valeria Mulli ${ }^{a}$, Dra. Marcela Caffulli ${ }^{a}$ y Dr. Julio Busaniche ${ }^{a}$
}

\section{RESUMEN}

Introducción. El subdiagnóstico y subregistro de sobrepeso y obesidad en pediatría es muy frecuente. El uso de una historia clínica electrónica podría contribuir favorablemente. Elobjetivo fue conocer el porcentaje de registro de este problema por pediatras de cabecera y analizar si se asociaba con la realización de estudios complementarios.

Métodos. Estudio de corte transversal. Se evaluó el registro del problema en pacientes pediátricos con sobrepeso y obesidad, y la presencia de resultados de glucemia, triglicéridos y colesterol de alta densidad en pacientes obesos.

Resultados. Se analizaron 7471 pacientes con sobrepeso y obesidad; el registro adecuado del problema fue del $19 \%$. El $44 \%$ de los obesos $(n=1957)$ tenía registro adecuado y el $32 \%$, resultados de laboratorio, con asociación significativa entre variables.

Conclusiones. Los porcentajes de registro de sobrepeso y obesidad y realización de estudios complementarios fueron bajos. El registro del problema se asoció a mayor solicitud de estudios.

Palabras clave: sobrepeso, obesidad, documentación, historia clínica electrónica.

http: / / dx.doi.org/10.5546/ aap.2020.132

Texto completo en inglés:

http: / / dx.doi.org/10.5546/ aap.2020.eng.132

Cómo citar: Silva C, Mulli V, Caffulli M, Busaniche J. Registro de sobrepeso y obesidad como problema de salud por pediatras de cabecera en una historia clínica electrónica. Arch Argent Pediatr 2020;118(2):132-135. a. Hospital Italiano de Buenos Aires e Instituto Universitario del Hospital Italiano de Buenos Aires, Ciudad Autónoma de Buenos Aires.

Correspondencia:

Dra. Carolina Silva: carolina.silvablanco@gmail.com

Financiamiento:

Ninguno.

Conflicto de intereses:

Ninguno que declarar.

Recibido: 16-1-2019

Aceptado: 9-9-2019

\section{INTRODUCCIÓN}

El sobrepeso y la obesidad constituyen una epidemia a nivel mundial, que afecta a alrededor de un tercio de los niños y adolescentes. ${ }^{1}$ El índice de masa corporal (IMC) permite realizar el diagnóstico en la práctica clínica y debe calcularse y percentilarse en forma anual en todos los mayores de 2 años. Un niño tiene sobrepeso cuando su IMC se encuentra entre los percentilos 85 y 97 para su edad y sexo, y obesidad, por encima del percentilo $97 .{ }^{2} \mathrm{El}$ exceso de peso implica un aumento en el riesgo presente y futuro de distintas patologías, en particular, el síndrome metabólico. Existe consenso respecto de la necesidad de pesquisar dislipidemia y diabetes en los niños obesos a través de la medición de glucemia, colesterol total, lipoproteínas de alta densidad (high-density lipoprotein-HDL-), lipoproteínas de baja densidad (low-density lipoprotein $-L D L-)$ y triglicéridos. ${ }^{3}$

El diagnóstico del sobrepeso y la obesidad depende de los médicos de cabecera. No obstante, muchos de estos niños y adolescentes no son identificados por sus pediatras. ${ }^{4,5}$ El registro de la obesidad como problema de salud es también subóptimo. ${ }^{6,7}$ Además, incluso en quienes son diagnosticados, no siempre se llevan a cabo la consejería y la solicitud de exámenes complementarios. ${ }^{8,9}$ El registro de los datos antropométricos en una historia clínica electrónica (HCE) puede contribuir a la identificación y al correcto seguimiento de los pacientes con sobrepeso y obesidad. ${ }^{10}$

Se desconoce el porcentaje de registro actual del sobrepeso y obesidad como problemas en nuestra población. El objetivo de este trabajo fue conocer el porcentaje de registro del sobrepeso y obesidad como problema de salud en la población pediátrica del Hospital Italiano de Buenos Aires (HIBA). Como objetivo secundario, se incluyó analizar si el registro del problema se asociaba con la realización de estudios de laboratorio recomendados en niños obesos. 


\section{MATERIALES Y MÉTODOS}

Estudio de corte transversal, entre julio de 2016 y junio de 2017. Se establecieron los siguientes criterios de inclusión: pacientes de ambos sexos de entre 2 y 19 años, atendidos en una consulta programada, con diagnóstico de sobrepeso u obesidad según el IMC, de acuerdo con el peso y la talla cargados en la HCE en los últimos 12 meses. Se excluyeron los datos de consultas no programadas y de médicos de cabecera no pertenecientes al Servicio de Clínica Pediátrica (médicos de familia u otras especialidades). Se consideró el IMC medido en la última consulta incluida en el período de análisis, y se usaron las referencias de la Organización Mundial de la Salud para definir sobrepeso y obesidad..$^{11}$

\section{Registro de sobrepeso/obesidad como problema}

De manera automática, se buscaron, entre los problemas de salud activos registrados en la HCE, los términos "sobrepeso" y "obesidad". Se tomó como registro adecuado la presencia de cualquiera de estos dos términos, sobre la base del apropiado reconocimiento del exceso de peso como problema de salud. Se calculó el porcentaje de registro en forma global y de cada médico de cabecera en forma individual.

\section{Análisis complementarios}

En la subpoblación de pacientes obesos (IMC mayor del percentilo 97) y pertenecientes al Plan de Salud del Hospital Italiano (PSHI), se midió el porcentaje de realización de estudios de laboratorio recomendados para la pesquisa de complicaciones asociadas. Para esto, se buscó la presencia en la HCE de resultados de glucemia, colesterol HDL y triglicéridos en los 12 meses previos y posteriores al diagnóstico de sobrepeso y/uobesidad.

Se incluyeron solo los pacientes obesos, ya que no existía consenso para la solicitud de estudios en los pacientes con sobrepeso. Los niños obesos con coberturas de salud distintas al PSHI no fueron considerados en este análisis para reducir la posibilidad de realización de los estudios de laboratorio fuera del ámbito del Hospital. Asimismo, solo se consideró la presencia de los tres valores de laboratorio, pues, de otra forma, el estudio podría tener un motivo distinto que la pesquisa de complicaciones asociadas a la obesidad.

Para evaluar la asociación entre el registro de obesidad como problema y la realización de exámenes de laboratorio para pesquisa de complicaciones, se realizó un análisis bivariado y se calculó chi ${ }^{2}$ con valor de p. Se utilizó el programa Stata versión 13.

\section{RESULTADOS}

Fueron incluidos 7471 pacientes con IMC mayor del percentilo 85 para edad y sexo, atendidos por 76 pediatras de cabecera. Del total de pacientes, el $19 \%$ tenía registro de sobrepeso/ obesidad como problema de salud (Figura 1).

Al analizar los datos de cada médico de cabecera, la mediana de registro adecuado fue del $14 \%$ (rango intercuartil del 9-25\%). De los 76 pediatras, 23 registraron el sobrepeso y la obesidad como problema de salud en el 0-10\% de sus pacientes; 27 , en el 10-20 \%; 11, en el 20-30\%; 8, en el $30-40 \%$, y 6 , entre el $40 \%$ y el $70 \%$ de sus pacientes. Siete de cada diez pediatras tuvieron una tasa de registro del $20 \%$ o menor, y solo el $5 \%$ habían registrado el sobrepeso u obesidad en, al menos, la mitad de sus pacientes.

Respecto de los pacientes obesos analizados $(\mathrm{n}=1957)$, el porcentaje de registro fue del $44 \%$, y el $32 \%$ tenían resultados de las tres determinaciones de laboratorio recomendadas (Figura 1). Un porcentaje mayor tenía resultados de glucemia, comparados con triglicéridos y colesterol HDL (el $40 \%$, el $35 \%$ y el $34 \%$, respectivamente). Se encontró una asociación estadísticamente significativa entre el registro del problema en la HCE y la presencia de resultados de laboratorio en la HCE $(p<0,01)$.

\section{DISCUSIÓN}

Los resultados de este trabajo muestran que, en nuestra población, se pierden muchas oportunidades de registro de sobrepeso y obesidad como problemas de salud en la consulta pediátrica. De forma similar a lo reportado en la bibliografía internacional, ${ }^{6-9}$ un porcentaje muy pequeño de los médicos evaluados registraron el diagnóstico de sobrepeso u obesidad en, al menos, la mitad de sus pacientes, cuando era deseable el registro cercano al $100 \%$. Esto puede deberse, en parte, al subdiagnóstico de estas enfermedades: si bien los niños son pesados y tallados en la consulta, y su IMC es calculado automáticamente en la HCE, es probable que los pediatras no determinen el percentilo de IMC en todos los pacientes. Esto último podría deberse a la falta de tiempo de las consultas, la falta de entrenamiento y las dificultades en el abordaje de esta patología. ${ }^{12}$ Sumado a lo anterior, la 
identificación correcta del problema de salud por el pediatra no siempre se traduce en un adecuado registro en la HCE.

En un estudio realizado en el HIBA en 20072008 ( $=14743)$, el $22 \%$ de los niños tenía sobrepeso y el $10 \%$, obesidad, y solo el $11 \%$ tenían un adecuado registro en la HCE. ${ }^{13} \mathrm{El}$ mayor porcentaje de registro observado en el presente estudio podría explicarse por las mejoras en la HCE en la última década y el creciente conocimiento sobre la obesidad y sus consecuencias.

Cabe destacar que el porcentaje de registro del problema de salud en los pacientes obesos fue superior comparado con los pacientes con sobrepeso. Se ha descrito que los niños con sobrepeso tienen más riesgo de no ser identificados, ${ }^{14}$ por lo que se pierde la posibilidad de intervenir tempranamente en este grupo. Al evaluar la correlación entre el registro y la presencia de determinaciones de laboratorio en los pacientes obesos, se encontró una asociación significativa. Esto refuerza la importancia de promover el adecuado diagnóstico y registro para mejorar el cumplimiento de las recomendaciones de pesquisa en este grupo.

Una de las fortalezas del trabajo es el elevado número de pacientes, en un centro donde la HCE está ampliamente implementada. Entre las debilidades, está la imposibilidad de diferenciar entre el subregistro y el subdiagnóstico de esta patología. Asimismo, al no buscarse términos en el texto libre, es posible que la proporción de pacientes adecuadamente diagnosticados por su pediatra sea mayor. Por último, la falta de resultados de laboratorio podría no responder a la ausencia de solicitud por su pediatra, sino a la baja adherencia de las familias.

Debería profundizarse el estudio de las causas del subregistro y las posibles estrategias de mejora. Los recordatorios en la HCE, la incorporación del gráfico de percentilos de IMC a esta y la información para padres podrían mejorar la identificación y el seguimiento de los niños con sobrepeso y obesidad. ${ }^{10,15}$

\section{CONCLUSIÓN}

El registro de sobrepeso y obesidad como problema por pediatras de cabecera en nuestra población fue bajo (el $19 \%$ ), así como la realización de estudios complementarios en niños obesos (el $32 \%$ ), a pesar del uso de una HCE. El adecuado registro del problema se asoció a una mayor pesquisa de complicaciones.

\section{REFERENCIAS}

1. Organización Mundial de la Salud. Informe de la Comisión para acabar con la obesidad infantil 2016. [Acceso: 14 de noviembre de 2018]. Disponible en: http:/ / apps.who.int/ gb/ebwha/pdf_files/WHA69/A69_8-sp.pdf.

2. Comité Nacional de Nutrición. Obesidad: guías para su abordaje clínico. Buenos Aires, 2016. [Acceso: 14 de noviembre de 2018]. Disponible en: https:/ / www.sap. org.ar/ uploads / consensos / obesidad-gu-iacuteas-parasu-abordaje-cl-iacutenico-2015.pdf.

3. Comité Nacional de Nutrición. Guías de práctica clínica para la prevención, el diagnóstico y el tratamiento de la obesidad. Arch Argent Pediatr. 2011; 109(3):256-66.

FIGURA 1. Registro de sobrepeso/obesidad como problema de salud y realización de estudios complementarios en niños obesos. Flujograma de pacientes analizados

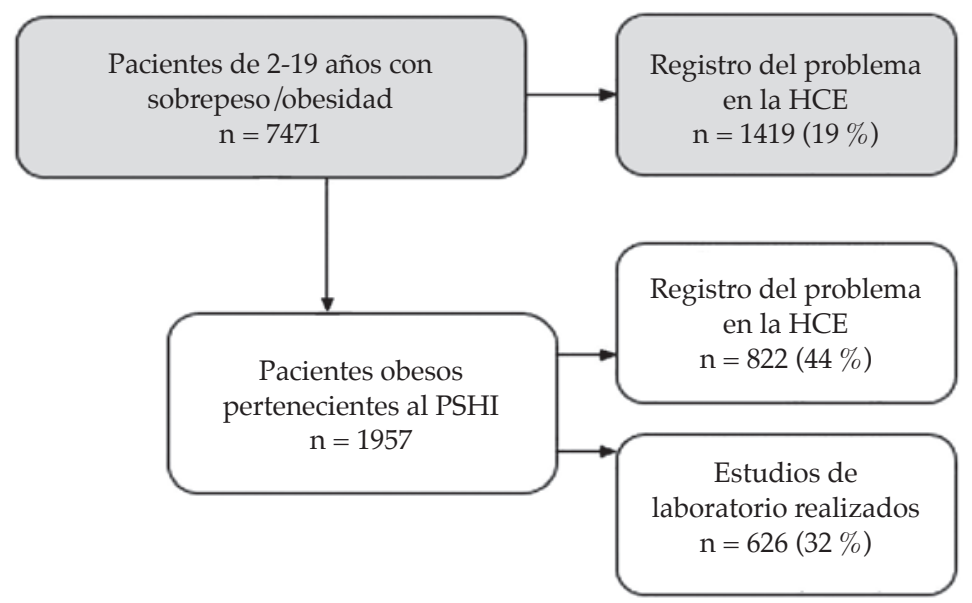

HCE: historia clínica electrónica; PSHI: Plan de Salud del Hospital Italiano. 
4. Huang JS, Donohue M, Golnari G, Fernandez S, et al. Pediatricians' weight assessment and obesity management practices. BMC Pediatr. 2009; 9:19.

5. Barlow SE, Dietz WH, Klish WJ, Trowbridge FL. Medical evaluation of overweight children and adolescents: reports from pediatricians, pediatric nurse practitioners, and registered dieticians. Pediatrics. 2002; 110(1 Pt 2):222-8.

6. Benson L, Baer HJ, Kaelber DC. Trends in the diagnosis of overweight and obesity in children and adolescents: 19992007. Pediatrics. 2009; 23(1):e153-8.

7. Patel AI, Madsen KA, Maselli JH, Cabana M, et al. Underdiagnosis of pediatric obesity during outpatient preventive care visits. Acad Pediatr. 2010; 10(6):405-9.

8. Dilley JK, Martin LA, Sullivan C, Seshadri R, et al. Identification of overweight status is associated with higher rates of screening for comorbidities of overweightin pediatric primary care practice. Pediatrics. 2007; 119(1):e148-55.

9. Benson LJ, Baer HJ, Kaelber DC. Screening for obesityrelated complications among obese children and adolescents: 1999-2008. Obesity. 2011; (19):1077-82.

10. Smith AJ, Skow Á, Bodurtha J, Kinra S. Health information technology in screening and treatment of child obesity: a systematic review. Pediatrics. 2013; 131(3):e894-902.
11. World Health Organization Multicentre Growth Reference Study Group. WHO Child Growth Standards: Length/ height-for-age, weight-for-age, weight-for-length, weightfor-height and body mass index-for-age: Methods and development. Geneva, 2006. [Acceso: 14 de noviembre de 2018]. Disponibleen:https: / / www.who.int/childgrowth/ standards/Technical_report.pdf.

12. Walker O, Strong M, Atchinson R, Saunders J, et al. A qualitative study of primary care clinicians' views of treating childhood obesity. BMC Fam Pract. 2007; 8:50.

13. Otero P, Duran P, Setton D, Eymann F, et al. Mismatch between the prevalence of overweight and obese children and adolescents and recording in electronic health records: a cross-sectional study. Inform Prim Care. 2011;19(2):75-82.

14. Cook S, Weitzman M, Auinger P, Barlow SE. Screening and counseling associated with obesity diagnosis in a national survey of ambulatory pediatric visits. Pediatrics. 2005; 116(1):112-6.

15. Keehbauch J, Miguel GS, Drapiza L, Pepe J, et al. Increased documentation and management of pediatric obesity following implementation of an EMR upgrade and education. Clin Pediatr (Phila). 2012; 51(1):31-8.

\title{
Daño renal agudo y cetoacidosis diabética en pacientes pediátricos: factores de riesgo
}

\author{
Acute kidney injury and diabetic ketoacidosis in pediatric patients: risk factors
}

\section{Dr. Carlos Sánchez García ${ }^{a}$ Dra. Mónica Briones Castellanos ${ }^{a}$ y Dr. Artemio Velasco Morales ${ }^{a}$}

\section{RESUMEN}

El daño renal agudo es causa de morbilidad en niños diabéticos en países en vías de desarrollo, especialmente, en pacientes con cetoacidosis diabética. El objetivo de esta investigación fue identificar factores de riesgo para daño renal agudo en pacientes con cetoacidosis diabética. Se realizó un estudio de cohorte retrospectiva. Se incluyeron 50 pacientes diabéticos con cetoacidosis; el $54 \%$ desarrollaron daño renal; en ellos, los niveles de glucosa y ácido úrico fueron mayores $(541 \mathrm{mg} / \mathrm{dl}$ contra $407 \mathrm{mg} / \mathrm{dl}, \mathrm{p}=0,014$, y $8,13 \mathrm{mg} / \mathrm{dl}$ contra $5,72 \mathrm{mg} / \mathrm{dl}, \mathrm{p}=0,015$, respectivamente). El ácido úrico mayor de $6,5 \mathrm{mg} / \mathrm{dl}$ demostró un odds ratio de $6,910(\mathrm{p}=0,027)$ para daño renal. En conclusión, la hiperuricemia fue un factor de riesgo para el desarrollo de daño renal agudo en estos pacientes. Son necesarios estudios prospectivos para determinar el papel del ácido úrico en la patogénesis del daño renal agudo en pacientes diabéticos. Palabras clave: cetoacidosis diabética, lesión renal aguda, pediatría, ácido úrico.

a. Hospital Niño de Saltillo. Saltillo, Coahuila, México.

Correspondencia:

Dr. Carlos Sánchez: sanchez.carlos8516@gmail.com

Financiamiento: Ninguno.

Conflicto de intereses: Ninguno que declarar.

Recibido: 26-3-2019

Aceptado: 16-9-2019 http: / / dx.doi.org/10.5546/ aap.2020.135

Texto completo en inglés:

http: / / dx.doi.org/10.5546/ aap.2020.eng.135

Cómo citar: Sánchez García C, Briones Castellanos M, Velasco Morales A. Daño renal agudo y cetoacidosis diabética en pacientes pediátricos: factores de riesgo. Arch Argent Pediatr 2020;118(2):135-138.

\section{INTRODUCCIÓN}

El daño renal agudo se define como el compromiso abrupto de la función renal, que lleva a desbalances hidroelectrolíticos, ácidobase y acumulación de productos de desecho. ${ }^{1}$ La incidencia se ha incrementado, especialmente, en los pacientes hospitalizados en estado crítico. ${ }^{2}$

Padecer diabetes mellitus es un factor de riesgo para desarrollar daño renal agudo, ya que se incrementa la vulnerabilidad renal a la isquemia. ${ }^{3} \mathrm{El}$ daño renal agudo es un predictor de morbilidad en los pacientes con diabetes mellitus tipo 1; incluso episodios leves se asocian con riesgo acumulado para desarrollar enfermedad renal crónica. ${ }^{4}$

La cetoacidosis diabética es una forma de presentación habitual en los pacientes 